\title{
Rehabilitation outcome in hemorrhagic transformation of ischemic stroke: a case presentation
}

Ioana Stanescu', Angelo Bulboaca ${ }^{1}$, Angela Ioana Cordosn, Rita Kallon, Adriana Bulboaca², Gabriela $\operatorname{Dogaru}^{3}$

Corresponding author: Bulboaca Adriana: e-mail : adriana_bulboaca@yahoo.com

1 University of Medecine and Pharmacy "Iuliu Hatieganu” Cluj, Neurosciences Department

${ }^{2}$ University of Medecine and Pharmacy "Iuliu Hatieganu” Cluj, Physiopathology Department

${ }^{3}$ University of Medecine and Pharmacy "Iuliu Hatieganu” Cluj, Physical Medicine and Rehabilitation Department ${ }^{n}$ Clinical Rehabilitation Hospital Cluj Napoca

\begin{abstract}
Hemorrhagic transformation (HT) may occur as a part of the natural history of acute ischemic stroke, with incidence between 13$43 \%$ in computer-tomography (CT) studies. Detection of HT in a patient with ischemic stroke is important in establishing treatment in the acute phase in assessing prognosis and in taking therapeutical decisions for secondary stroke prevention. There were controversies regarding optimal treatment in acute ischemic stroke with HT, especially if the patient needs further anticoagulant or antiplatelet treatment. Rehabilitation treatment needs to be continued for post-stroke patients who developed HT, because HT does not decrease rehabilitation outcome. We present rehabilitation treatment and prognosis in a case of hemorrhagic transformation after an embolic ischemic stroke treated with anticoagulants, which required reinitiation of anticoagulant treatment.
\end{abstract}

Key words: acute ischemic stroke, hemorrhagic transformation, anticoagulant treatment, rehabilitation prognosis

\section{Introduction}

Hemorrhagic transformation (HT) may occur as a part of the natural history of acute ischemic stroke or may be precipitated by the use of thrombolytic, anticoagulant or antiplatelet treatment [1]; is defined as extravasation of blood components through a damaged blood-brain barrier on an area of ischemic infarct, confirmed by computer-tomography (CT) imaging [2]. Bleeding occurrence has variable aspects, from petechial heterogeneous hyperdensity to true parenchimal hematoma.

The incidence of spontaneous HT ranges from 38\% to $71 \%$ in autopsy studies and from $13 \%$ to $43 \%$ in CT studies of patients with cerebral infarction $[3,4,5]$. A study performed by Japanese authors which included only patients with ischemic stroke admitted in a Rehabilitation facility within 2 months from stroke onset, found an incidence of $18.2 \%$ of HT [6].

Detection of HT is done routinely by native CT scan. Magnetic resonance imaging (MRI) with T2* weighted gradient echo sequences has a higher rate of detection of HT, but with heterogenous results [7]. MRI-based studies reported incidences of early HT between 46.3 and $51.6 \%$ [8,9].

Detection of HT in a patient with ischemic stroke is important is assessing prognosis and in taking therapeutical decisions in the acute phase and for secondary stroke prevention.
There were controversies regarding optimal treatment in acute ischemic stroke with hemorrhagic transformation on CT scan, especially if the patient needs further anticoagulant or antiplatelet treatment. For this group of patients, recent guidelines recommend that initiation or continuation of antiplatelet or anticoagulation therapy may be considered, depending on the specific clinical scenario and underlying indication [10], but individual assessment of the clinical indication, benefits, and associated risks is warranted [5, 11].

Rehabilitation treatment can be continued for patients who have already developed HT at the time of transfer to a rehabilitation hospital in the acute phase of cerebral infarction, but careful observation of the symptoms and CT scans over a defined period must be maintained [6]. Hemorrhagic transformation of an ischemic lesion does not affect rehabilitation outcome in stroke survivors [12].

The aim of this paper is presentation of therapeutical challenges and rehabilitation prognosis in a case of hemorrhagic transformation in an embolic ischemic stroke treated with anticoagulants, which also required reinitiation of anticoagulant treatment. 


\section{Method: case presentation}

We present the case of a 68 years old woman, living in rural area, which suffered 12 days before admission to Rehabilitation Hospital an acute ischemic stroke in the right middle cerebral artery (MCA) territory, and was transferred from a Regional Neurological Clinic. The patient had a history of poorly controlled diabetes mellitus, treated with insulin, of treated arterial hypertension, hypercholesterolemia and ischemic cardiopathy. During her hospitalization for acute ischemic stroke, atrial fibrillation was diagnosed and anticoagulant treatment with a direct oral anticoagulant agent (DOAC) was initiated.

Clinical examination at admission showed an obese patient (BMI = $35 \mathrm{~kg} / \mathrm{m} 2)$, with complete arrhythmia (atrial fibrillation) with 110-120 b/min and BP values of 130/80 mmHg. Neurological examination showed a somnolent patient, with oculogyric deviation toward the right side, left flaccid hemiplegia with motor deficit of 0/5 at Medical Research Council (MRC) scale at upper and lower limbs, left Babinski sign, leftsided exteroceptive hypoestesia and inattention, sphincterian incontinence, with a NIHSS score of 25 points, indicating a severe stroke.

Modified Rankin score (mRS) at admission was 5 points, indicating a severe disability.

Blood tests at admission showed hyperglycemia. Renal function, hepatic function were in normal ranges, and also platelet count was normal. Doppler ultrasonography of carotid and vertebral arteries showed atheromatous plaques, without significant stenosis.

Patient treatment at admission in rehabilitation facility included insulin, antiarrythmic (digoxin, metoprolol), antihypertensive (candesartan) and statin. Direct anticoagulant treatment was initiated 5 days ago. No external bleeding signs were noted.

The second day after admission in Rehabilitation, the patient complained of headache and continued to be somnolent.

A native cerebral CT scan was performed, showing ischemia in the right superficial MCA territory with hemorrhagic transformation (petechial hyperdensities without space-occupying effect), corresponding to type 1 hemorrhagic infarctus (HT1) - Figures 1 and 2.

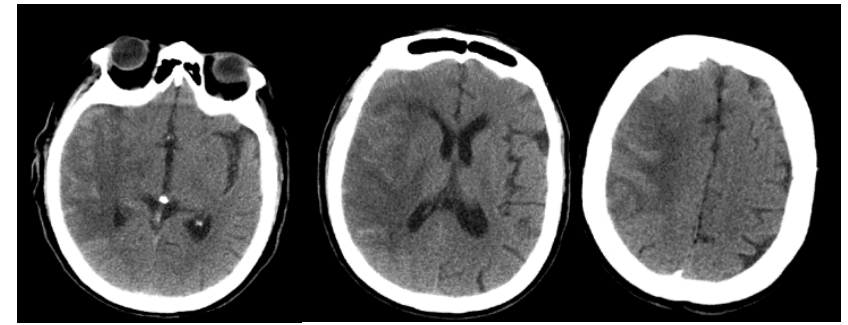

Figure 1: Axial native CT scan showing petechial hyperdensities on the R MCA territory

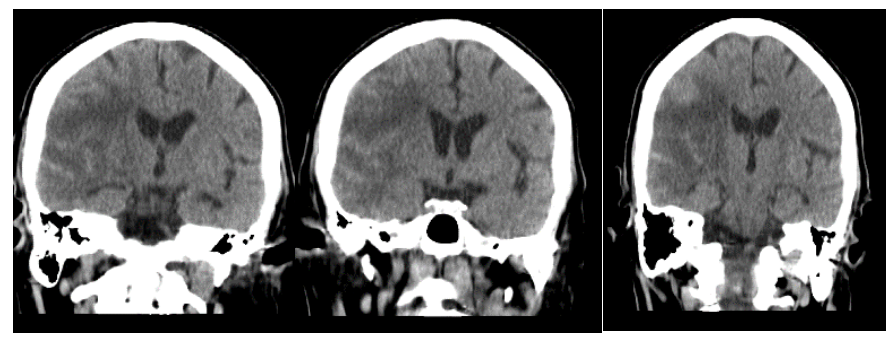

Figure 2: Coronal native CT scan showing petechial hyperdensities on the R MCA territory

The therapeutical decision was for interrupting anticoagulant treatment; enoxaparin in prophylactic doses for prevention of deep venous thrombosis (DVT) was started the third day after admission. Blood pressure control was achieved, with systolic BP values maintained less than $140 \mathrm{mmHg}$, in order to maintain cerebral perfusion and to avoid further bleeding [1].

The clinical evolution was favourable, headache disappeared two days after interruption of DOAC treatment, while the patient has become more alert and cooperant. At this point, rehabilitation treatment was started, initially with kinesitherapy, aimed to passive mobilization and postural exercises.

The cardiological examination confirmed permanent atrial fibrillation and recommended chronic anticoagulation treatment after resorbtion of the hemorrhagic petechiae.

After 7 days of withdrawal of anticoagulant treatment, patient's neurological status improved: the patient was alert, without headache. Follow-up cerebral CT scan (not shown) showed complete resorbtion of the hemorrhagic petechiae. Our patient with atrial fibrillation had a CHADS2-VAsc score of 6 points (13.6\% per year risk of stroke/transient ischemic attack (TIA)/systemic embolism) [13] and a HAS-BLED score of 3 points. Anticoagulant treatment was restarted, and, again, a DOAC was chosen because it carries a lower risk of intracerebral bleeding compared with vitamin $\mathrm{K}$ antagonists (VKA). 
The DOAC dose was the lowest efficient dose for stroke prevention. Enoxaparin treatment was stopped concomitant with restarting DOAC.

At the end of the rehabilitation program of 21 days, after an intense program of passive exercises, posturing, strengthening exercises, functional electrical stimulation of lower limb muscles, motor deficit showed mild improvement at lower limb (quotation 2/5 on MRC scale). NIHSS score at discharge is 16 points (have improved with 5 points). Modified Rankin score also improved to 4 points at discharge. Patient's quality of life showed significant changes after rehabilitation treatment [14]. Further sessions of rehabilitation treatment in an outpatient facility and in a balnear resort were planned, aimed to improve pateint's quality of life [15].

For presentation of this case, patient's family consent and Hospital Ethics Committee advice were obtained.

\section{Results:}

This patient with vascular risk factors suffered an massive ischemic stroke in the right MCA territory; the etiology of stroke was presumed to be embolic, based on the concomitant presence of atrial fibrillation at stroke onset. Hemorrhagic transformation has become symptomatic in the 14-th day after stroke onset, manifested by headache and alteration in consciousness. Risk factors for hemorrhagic transformation in this case were large area of infarctus, high NIHSS score, embolic stroke with atrial fibrillation and hyperglycemia in a poorlycontrolled diabetic patient. We consider that early introduction of anticoagulant treatment was a decisive factor in bleeding occurence in the ischemic area.

Differential diagnosis in this case was made with (a): extension of the infarctus (but the dimensions of the ischemic area were not changed comparative to CT scan obtained 7 days after stroke), (b): secondary parenchimal hematoma (no homogenous bleeding and no mass effect appeared on our CT scan) and (c) infection with sepsis which could mimic neurological deterioration (no fever, no leucocytosis, no modified inflammatory parameters were present).

\section{Discussion:}

Hemorrhagic transformation (HT) of an acute ischemic stroke is defined as extravasation of blood components through a damaged blood-brain barrier which produces bleeding in the affected cerebral area. Depending on the amount of bleeding detected on native cerebral CT scan, HT is classified into 2 subtypes: hemorrhagic infarctus (petechial hyperdensities without space-occupying effect on CT scan) and parenchimal hematoma (homogenous bleeding with mass effect) [2]. Hemorrhagic infarctus is further classified in 2 subtypes: small hyperdense petechiae (HT1) and confluent hyperdensities without mass effect throughout the infarct zone (HT2) [2]. Parenchimal hematoma, associated with worsening of the NIHSS by at least 4 points [16] is classified in 2 subtypes: PH1 parenchymal hematoma which involved less than $30 \%$ of the ischemic territory with minimal mass effect and PH2 - parenchymal hematoma of more or equal to $30 \%$ of the infarct with obvious mass effect [17].

Another classification is based on the timing when HT is noticed: early HT - in patients who developed HT within 1 week and delayed HT - in more than 1 week after stroke onset [6]. The etiopathogenetic mechanisms are different between the 2 groups: early $\mathrm{HT}$ is due to reperfusion (when the clot that blocked arterial blood flow to the brain is fragmented), and delayed HT is mainly due to abnormal vascular permeability and increase in blood flow, after decrease of cerebral edema.

A more practical classification divides hemorrhagic transformation after ischemic stroke in asymptomatic and symptomatic [18].

Predictors for HT are massive area of infarction, gray matter involvment, atrial fibrillation with cerebral embolism, high NIH Stroke Scale (NIHSS) score, hyperglycemia, lower LDL- and total cholesterol levels, lower platelet count, use of fibrinolytic treatment, endovascular treatment, poor collateral vessels, early CT signs of ischemia and hyperdense middle cerebral artery sign $[3,19]$. 
Cerebral microbleeds (CMBs) do not predict the presence of hemorrhagic transformation in acute ischemic stroke patients with $\mathrm{AF}$ and/or rheumatic heart disease. The results were not affected by anticoagulant or antiplatelet agents used in acute stage of ischemic stroke [20].

The prognosis of patients with hemorrhagic infarcts remain under debate. Hemorrhagic transformation (HT) following acute cerebral ischemia has been postulated to exert a negative influence on the early clinical course and outcome [9]. In a study which used the database of patients recruited in the European Cooperative Acute Stroke Study I (ECASS I), authors showed that patients exhibiting an early (within 36 hours from stroke onset) hemorrhagic transformation did not a have a higher risk of neurological deterioration compared with patients without hemorrhagic transformation; also, 3-month mortality and disability were also not influenced by HT [20].

Paciaroni et al. concluded that only massive hemorrhagic transformation with parenchymal hematoma (incidence in $3 \%$ of patients) is associated with unfavorable prognosis. Predictors for parenchymal hematoma in this study were large lesions, cardioembolic stroke, high blood glucose, and thrombolytic treatment [21].

Another study conducted by Bayramoğlu et al. showed that there was no association between HT development and functional prognosis, although HT was detected in $32.2 \%$ of patients with cerebral infarction who were admitted to a rehabilitation hospital in the acute phase. Tan et al. included in their study only ischemic stroke patients not receiving thrombolytic treatment; they showed that spontaneous HT was not independently associated with a worse 3-month prognosis for ischemic stroke [19].

Therapeutical management in ischemic stroke with HT is a delicate issue, especially in patients who received thrombolytic treatment or in patients which need early anticoagulation.

Fibrinolitic treatment with rt-PA was incriminated for increased incidence of HT in treated patients, especially in large strokes. Latest 2018 Guidelines for the Early Management of Patients With Acute Ischemic Stroke stated that despite increased risk of hemorrhagic transformation in patients with severe stroke symptoms, there is still proven clinical benefit of IV thrombolysis [10].
Another study found that there is no increased risk of hemorrhagic transformation even with early initiation of antiplatelet or anticoagulant therapy (less than 24 h) following IV alteplase or endovascular therapy compared to initiation after $24 \mathrm{~h}$, and concluded that early (within 24 hours after initiation) antithrombotic administration did not increase hemorrhages after recanalization treatment. [22]

Regarding initiation of anticoagulant treatment, same 2018 Guidelines for the Early Management of Patients With Acute Ischemic Stroke recommend for most patients with an acute ischemic stroke in the setting of atrial fibrillation, that it is reasonable to initiate oral anticoagulation within 4 to 14 days after the onset of neurological symptoms [10].

For the category of patients with hemorrhagic transformation after acute ischemic stroke, numerous observational studies suggest that antithrombotics can be safely initiated or continued, with individual assessment of the clinical indication, benefits, and associated risks $[5,11]$. In the 2018 Guidelines there is a Class IIb, level B-NR recommendation that initiation or continuation of antiplatelet or anticoagulation therapy may be considered, depending on the specific clinical scenario and underlying indication [10].

There are few data concerning efficacy and outcome of rehabilitation treatment after HT. Rehabilitation can be continued for patients who have already developed HT at the time of transfer to a rehabilitation hospital in the acute phase of cerebral infarction, but careful observation of the symptoms and CT scans over a defined period must be maintained [6]. Hemorrhagic transformation of an ischemic lesion does not affect rehabilitation outcome in stroke survivors [12].

\section{Conclusions:}

Hemorrhagic transformation is frequently observed on control CT scans of patients with acute ischemic strokes, with or without thrombolytic treatment. Symptomatic HT appears if there is extravasation of a large amount of blood, producing a mass effect. Incidence of HT is greater in large and severe strokes, especially of cardio-embolic etiology. The presence of HT does not seem to interfere with clinical evolution and early prognosis of stroke patients (except those with parenchimal hematoma). 
Initiation or continuation of anticoagulation therapy in patients with HT of ischemic stroke may be considered, according to recent guidelines, but only based on individual decision. Rehabilitation treatment can be continued if hemorrhagic transformation occurs in a stroke patient.

Our case reflects hemorrhagic transformation occurring in a typical cardio-embolic stroke. Despite the initial negative influence of anticoagulant treatment, clinical evolution of the patient was favorable, with improvement of neurological status. The favorable early outcome made possible initiation of rehabilitation therapy and of anticoagulant treatment, at a lower efficient dose, with good prognosis at patient's discharge.

\section{BIBLIOGRAPHY:}

1. Stone JA, Willey JZ, Keyrouz S, Butera J, McTaggart RA, Cutting S, Silver B, Thompson B, Furie KL, Yaghi S. Therapies for hemorrhagic transformation in acute ischemic stroke. Curr Treat Options Neurol. 2017;19:1.

2. Fiorelli M, Bastianello S, von Kummer R, del Zoppo GJ, Larrue V, Lesaffre E, Ringleb AP, Lorenzano S, Manelfe C, Bozzao L. Hemorrhagic transformation within 36 hours of a cerebral infarct: relationships with early clinical deterioration and 3-month outcome in the European Cooperative Acute Stroke Study I (ECASS I) cohort. Stroke. 1999;30:2280-2284]

3. Jie Zhang,Yi Yang, Huijie Sun,Yingqi Xing. Hemorrhagic transformation after cerebral infarction: current concepts and challenges. Ann Transl Med. 2014 Aug; 2(8): 81]

4. Terruso V, D'Amelio M, Di Benedetto N, Lupo I, Saia V, Famoso G, Mazzola MA, Aridon P, Sarno C, Ragonese P, Savettieri G. Frequency and determinants for hemorrhagic transformation of cerebral infarction. Neuroepidemiology. 2009;33:261-265

5. Kim JT, Heo SH, Park MS, Chang J, Choi KH, Cho KH. Use of antithrombotics after hemorrhagic transformation in acute ischemic stroke. PLoS One. 2014;9:e89798. doi: 10.1371/journal.pone.0089798.

6. Maeshima S, Okamoto S, Okazaki H, Mizuno S, Asano N, Tsunoda T, Maeda H, Masaki M,
Sonoda S. Hemorrhagic Transformation in Patients with Cerebral Infarction Referred to a Rehabilitation Hospital. Interv Neurol. 2016 Mar; 4(3-4): 69-74

7. Mayer TE, Schulte-Altedorneburg G, Droste DW, Brueckmann H: Serial CT and MRI of ischemic cerebral infarcts: frequency and clinical impact of haemorrhagic transformation. Neuroradiology 2000; 42: 233-239

8. Hjort $\mathrm{N}, \mathrm{Wu} \mathrm{O}$, Ashkanian $\mathrm{M}$, Solling $\mathrm{C}$, Mouridsen K, Christensen S, Gyldensted C, Andersen G, Ostergaard L: MRI detection of early blood-brain barrier disruption: parenchymal enhancement predicts focal hemorrhagic transformation after thrombolysis. Stroke 2008; 39: 1025-1028.

9. Kimura K, Iguchi Y, Shibazaki K, Kobayashi K, Uemura J, Aoki J, Yamashita S, Terasawa Y, Matsumoto N: Recanalization between 1 and 24 $\mathrm{h}$ after t-PA therapy is a strong predictor of cerebral hemorrhage in acute ischemic stroke patients. J Neurol Sci 2008; 270: 48-52.

10. William J. Powers, Alejandro A. Rabinstein, Teri Ackerson, Opeolu M. Adeoye, Nicholas C. Bambakidis, Kyra Becker, José Biller, Michael Brown, Bart M. Demaerschalk, Brian Hoh Edward C. Jauch, Chelsea S. Kidwell, Thabele M. Leslie-Mazwi, Bruce Ovbiagele, Phillip A. Scott, Kevin N. Sheth, Andrew M. Southerland, Deborah V. Summers and David L. Tirschwell. 2018 Guidelines for the Early Management of Patients With Acute Ischemic Stroke: A Guideline for Healthcare Professionals From the American Heart Association/American Stroke Association. Stroke. 2018;49:e46-e110; originally published online January 24, 2018;

11. England TJ, Bath PM, Sare GM, Geeganage C, Moulin T, O’Neill D, Woimant F, Christensen H, De Deyn P, Leys D, Ringelstein EB; on behalf of the TAIST Investigators. Asymptomatic hemorrhagic transformation of infarction and its relationship with functional outcome and stroke subtype: assessment from the Tinzaparin in Acute Ischaemic Stroke Trial. Stroke. 2010;41:2834-2839.

12. Bayramoğlu M, Karataş M, Leblebici B, Cetin N, Sözay S, Turhan N. Hemorrhagic transformation in stroke patients. Am J Phys Med Rehabil. 2003;82:48-52 
13. Friberg L, Rosenqvist M, Lip GY. Evaluation of risk stratification schemes for ischaemic stroke and bleeding in 182678 patients with atrial fibrillation: the Swedish Atrial Fibrillation cohort study. Eur Heart J. 2012 Jun;33(12):1500-10.

14. Dogaru Gabriela, Ispas Alexandra, Bulboacă Adriana, Motricală Marieta, Stănescu Ioana. Influence of balnear therapy at Băile Tușnad on quality of life of post-stroke patients. Balneo Research Journal. 2017;8(4):201-205

15. Dogaru Gabriela, Ispas Alexandra, Stănescu Ioana, Motricala Marieta, Ákos Molnar. A clinical study on the efficacy of natural therapeutic factors in Băile Tuşnad for the rehabilitation of post-stroke patients. Balneo Research Journal. 2017;8(1):5-10.

16. Liu K, Yan S, Zhang S, Guo Y, Lou M. Systolic blood pressure variability is associated with severe hemorrhagic transformation in the early stage after thrombolysis. Transl Stroke Res. 2016;7:186-191.

17. Hacke W, Kaste M, Fieschi C, von Kummer R, Davalos A, Meier D, et al. Randomised doubleblind placebo controlled trial of thrombolytic therapy with intravenous alteplase in acute ischaemic stroke (ECASS II). Lancet. 1998;352:1245-51.

18. The NINDS t-PA Stroke Study Group . Intracerebral hemorrhage after intravenous t-PA therapy for ischemic stroke. Stroke.1997; 28, 2109-2118

19. Tan S, Wang D, Liu M, Zhang S, Wu B, Liu B. Frequency and predictors of spontaneous hemorrhagic transformation in ischemic stroke and its association with prognosis. J Neurol. 2014 May;261(5):905-12.

20. Liu J, Wang D, Li J Lin J, Xiong Y, Liu B, Wei C, Wu B, Ma Z, Zhang S, Liu M. Cerebral Microbleeds Do Not Predict Hemorrhagic Transformation in Acute Ischemic Stroke Patients with Atrial Fibrillation and/or Rheumatic Heart Disease. Curr Neurovasc Res. 2017;14(2):104-109. doi: 10.2174/1567202614666170313111251

21. Paciaroni M, Agnelli G, Corea F, Ageno W, Alberti A, Lanari A, Caso V, Micheli S, Bertolani L, Venti M, Palmerini F, Biagini S, Comi G, Previdi P, Silvestrelli G. Early hemorrhagic transformation of brain infarction: rate, predictive factors, and influence on clinical outcome: results of a prospective multicenter study. Stroke. 2008;39:2249-2256.

22. Han-Gil Jeong, Beom Joon Kim, Mi Hwa Yang, et al. Stroke outcomes with use of antithrombotics within 24 hours after recanalization treatment. Neurology; 2016;87:17 